\title{
Transversal Symmetry Breaking in Novel Photonic Crystal Waveguide: Innovative Manner to Master Defect Band Dispersion Relation
}

\author{
Moïse Sotto ${ }^{1}$, Kapil Debnath ${ }^{1}$, Muhammad Khaled Hussain ${ }^{1}, \mathrm{Zuo} \mathrm{Li}^{1}$, \\ Fayong Liu ${ }^{1}$, Ali Zarrar Khokhar ${ }^{1}$, Shinichi Saito ${ }^{1 *}$, \\ ${ }^{1}$ Nanoelectronics \& Nanotechnology Research Group, Electronics and Computer Science, Faculty of Physical Sciences and \\ Engineering, University of Southampton Building 53, SO17 1BJ, UK. E-mail: S.Saito@soton.ac.uk
}

\begin{abstract}
We present a novel Photonic Crystal Slab (PhC) fabricated in Silicon (Si) with pattern edge along $<11-2>$ direction. Such a design enabled a dry and wet etching processes combination to atomically flatten the sidewall surface defined by Si[111]. Moreover, a new method to tailor the relation dispersion of line defect $\mathrm{PhC}$ waveguide (W1) using this structure is introduced. By mismatching the upper and lower part of the waveguide in the propagation direction, FDTD simulations demonstrate a net shift of the Cut-Off wavelength from $1605 \mathrm{~nm}$ to $1742 \mathrm{~nm}$ for a mismatch of zero and half of the lattice constant of the crystal a, respectively. We discuss both use of this new PhC for repeatable photonic device on-chip integration and the benefit of this new waveguide design method to engineer large bandwidth mode.
\end{abstract}

\section{Introduction}

Despite the fact that PhCs are promised to a broad range of applications, their availability is untapped by their fabrication repeatability. The challenge is to overcome underlying fluctuations in the design due to submicron lithography technology preventing their inclusive integration on chip. Here we demonstrate the fabrication of a $\mathrm{PhC}$ reducing surface roughness by the use of TetraMethylAmmonium Hydroxide (TMAH) anisotropic wet etching post-processing already developed for loss reduction in slot waveguide [1], and its potential in PhC waveguide mode dispersion engineering with an original method which consist to mismatch the upper and lower part of the $\mathrm{PhC}$ waveguide. The transversal mirror symmetry breaking is pointed out by a design parameter so-called

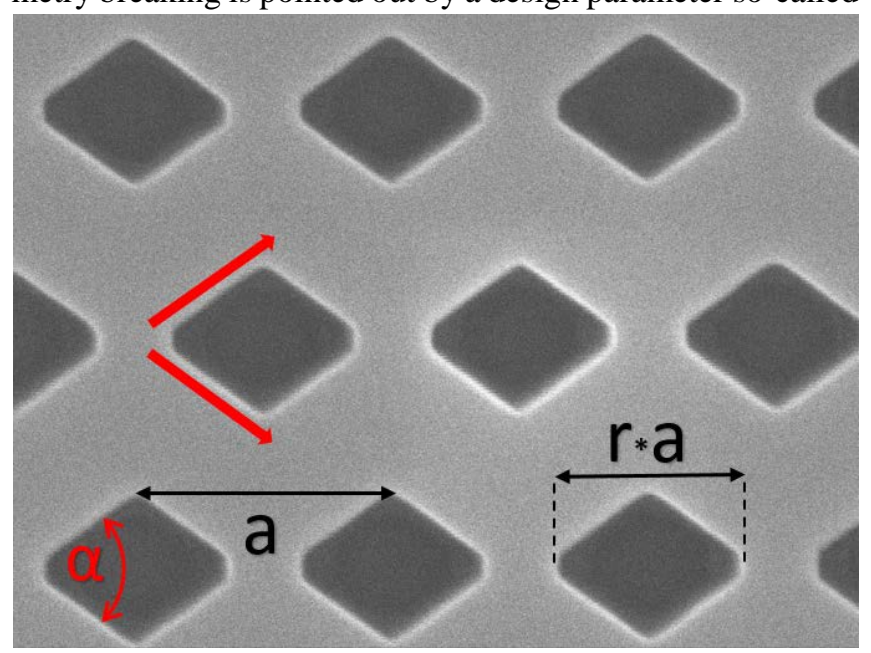

Fig. 1 SEM image of the fabricated triangular PhC slab with highlighted parameters: a the lattice constant $405 \mathrm{~nm}, \mathrm{r}=0.7, \alpha$ the angle between [111] planes in red and the $<11-2>$ directions displayed with red arrows.

the mismatch shifting them in the propagation direction. We extracted from the FDTD simulations, the relevant photonic property of the band defect mode, the normalized dispersion relation. The photonic waveguide characteristics display a clear confinement transition in the dispersion curve by pinning the band edge at a much lower energy at the end of the Brillouin Zone for the maximum mismatch of half of a period compared to the unmodified waveguide.

\section{Atomically Flat Sidewall Surface PhC}

We fabricated a triangular PhC slab with the pattern edge aligned along $<11-2>$ direction (Fig. 1) on a Silicon-On-Insulator (SOI) substrate with $220 \mathrm{~nm}$ thick layer of Si grown along $\langle 110\rangle$ direction on $2 \mu \mathrm{m}$ thick buried oxide (BOx). The pattern is defined by an equilateral rhombus with a lower angle of $\alpha=70.53^{\circ}$, an intrinsic angular separation between [111] planes and the longer diagonal proportional to the lattice constant by a ratio $r$ highlighted on the Scanning Electron Microscope (SEM) imaging performed after fabrication (Fig. 1). The fabrication process [1] started by annealing the SOI substrates in order to thermally grow a 20nm thick layer of $\mathrm{SiO}_{2}$ on top, then spin coating 260-nm thick ZEP-520A positive ebeam resist layer. The subsequent e-beam lithography was continued by a dry etching process in an Inductively Coupled Plasma (ICP) etcher to etch down the pattern into the top oxide and $\mathrm{Si}$ layer pursued by the removal of the remaining resist in an $\mathrm{O}_{2}$ plasma asher. After fuming nitric acid and diluted hydrofluoric acid treatments for cleaning purposes, the sam-

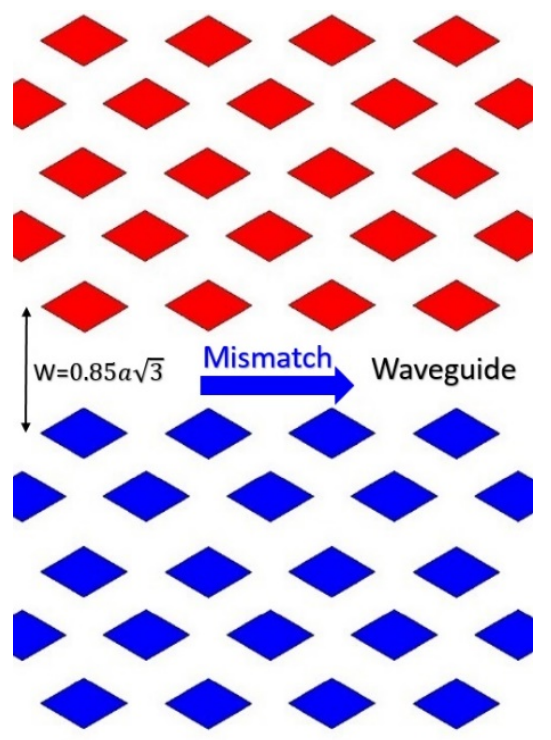

Fig 2. Mismatched PhC Geometry The lower part (blue) is displaced with respect to the upper part (red) along the propagation axis. ple was immersed in $25 \%$ aqueous solution of TMAH for 10 minutes at room temperature. Taking into account the anisotropic wet etching and the Si exposure of only sidewall surface due to the $\mathrm{SiO}_{2}$ hard mask protection on top and the $\mathrm{BOx}$ underneath, this step principally results in a drastic reduction of the surface roughness induced by the very slow etch rate normal to the $\mathrm{Si}$ [111] plane. Thereupon, another longer 
diluted hydrofluoric acid treatment was applied to the sample to suspend the Si membrane by removing the $\mathrm{BOx}$ and the $\mathrm{SiO} 2$ top layer.

To form a bandgap around the telecommunication wavelength $\lambda=1550 \mathrm{~nm}$, by considering the slab thickness and the refractive index of $\mathrm{Si}$ in this range $\mathrm{n}=3.477$, design parameters were calculated to be $a=405 \mathrm{~nm}$ and $r=0.7$. The SEM image of the fabricated PhC lab (Fig. 1) shows a smooth boundary of the pattern indicating a vertical interface with very low surface roughness. However, the edge between [111] planes is rounded due to the establishment of metastable surfaces at the corner, providing the opportunity to study the robustness of this metastable surface to a longer anisotropic wet-etching and, in the future, state on the ineluctability or not of this artefact.

\section{Innovative PhC Waveguide Mode Shaping by Trans- versal Mirror Symmetry Breaking}

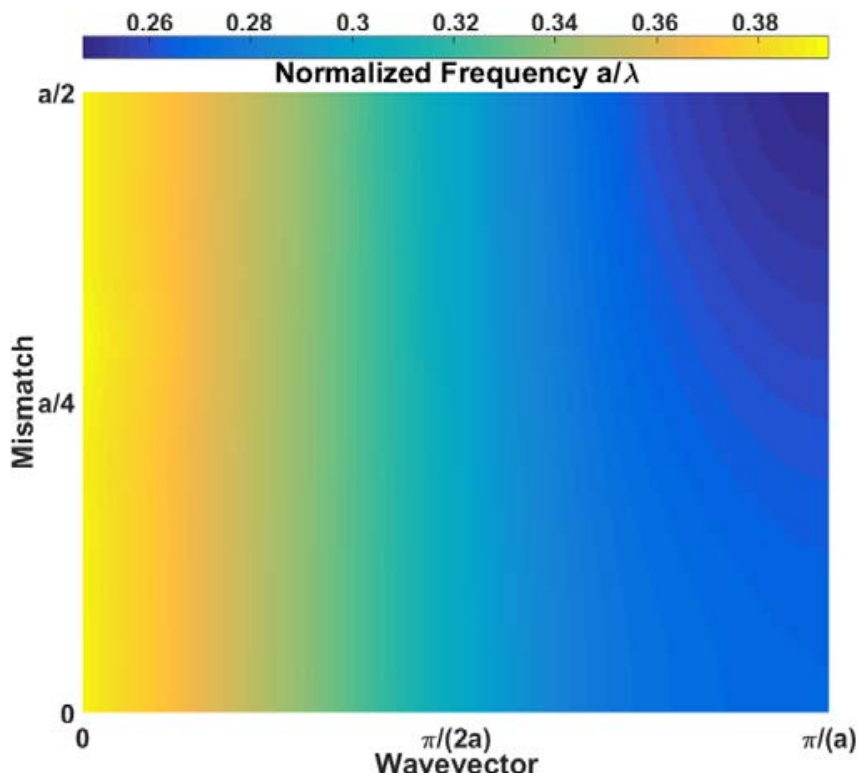

Fig 3: Normalized dispersion relation of the waveguide mode in the Brillouin Zone in function of the Mismatch, the shifting of the lower part of the $\mathrm{PhC}$ waveguide in the propagation direction.

It is well known that the support of a waveguide mode in a $\mathrm{PhC}$ line defect is determined by index and/or gap guiding. Since symmetries [2] and guiding mechanisms [3] play a key role in bands repulsion. We propose here an innovative method to alter the dispersion of a line defect by mismatching in the propagation direction the crystal on both sides of the waveguide, in the framework of our novel PhC structure (Fig. 2). Until today, the methods proposed to engineer $\mathrm{W} 1$ dispersion relation consist of symmetric changes of the structure such as modifying the holes size around the defect [4] or the position of particular lines on both sides of the waveguide [5]. Instead of symmetrically perturbing the crystal around the defect, our approach breaks the transversal mirror symmetry of the defect line. Consequently, the mismatch changes the electric field disposition forcing the mode to access elusive energy values in the unmodified structure. First, we chose the lattice constant and the ratio of the PhC to be $a=405 \mathrm{~nm}$ and $r=0.7$, respectively. Another important feature of the design is that the line defect width $\mathrm{W}$ had been squeezed decreasing the upper and lower part separation to $0.85 \sqrt{3} a$. Then, we focused our study on the initial band defect mode escaping from the air band. The relation dispersion dependences on the mismatch are summarized in Fig. 3. The mismatch effect on the mode is to dip the energy level at the end of the Brillouin zone transforming a constant dispersion curve of the mode around this range of wave-vector into a sloped band. This alteration reflects an unexpected transformation of the guidance mechanism. If the wave-vector independence of the waveguide mode reflects a bandgap guiding of the mode, the slope of the band is the signature of the index guiding. In other words, the mismatch between both sides of the waveguide progressively forces the mode to rely on the index confinement rather than the $\mathrm{PhC}$ gap effect. This new method can be also used to control photonic interaction between defect modes bands in the gap region by fine-tuning their physical guiding nature.

Indeed, in our study, this transition of behavior regarding the mode confinement is expressed by the lowering of the energy minimum, as it is shown on Fig. 3 . Leading to a broader range of operability of the waveguide mode such as the upper cut-off wavelength has been shifted from $1605 \mathrm{~nm}$ to 1742 $\mathrm{nm}$, for a mismatch of $0 \mathrm{~nm}$ and $a / 2$, respectively.

\section{Conclusions}

In this paper, we presented a new design of $\mathrm{PhC}$ with atomically flat surface based on a pattern with edges aligned with Si[111] planes allowing a wet etching process acting to remarkably reduce the surface roughness. Therefore, increasing the accuracy and the reproducibility of the design, paving the way for on chip integration of high performant and reproducible Si PhC-based technology.

In addition, we discussed a unique way to engineer the line defect waveguide mode in PhCs to increase the frequency range operability by tailoring the band shape of the mode relying on a transition of the guiding mechanism and demonstrate the capability of our novel structure to demonstrate such surprising functions.

\section{Acknowledgements}

This work is supported by EPSRC Standard Grant (EP/M009416/1), EPSRC Manufacturing Fellowship (EP/M008975/1), EPSRC Platform Grant (EP/N013247/1), and EU FP7 Marie-Curie Carrier-Integration-Grant (PCIG13-GA-2013-618116). The data from the paper can be obtained from the University of Southampton ePrint research repository: http://doi.org/10.5258/SOTON/D0096

\section{References}

[1] K. Debnath et al Front. Mater. 351 (2016)

[2] M. Ibanescu et al Phys. Rev. Lett. 92, 063903 (2004)

[3] M. Notomi et al Phys. Rev. Lett. 87, 253902 (2001).

[4] L. H. Frandsen, et al Opt. Express 14, 9444-9450 (2006).

[5] J. Li et al Opt. Express 16, 6227-6232 (2008). 